\title{
Fetal Magnetic Resonance Imaging of Normal Spinal Cord: Evaluating Cord Visualization and Conus Medullaris Position by T2-weighted Sequences
}

\author{
Yen-Ling Huang ${ }^{1}$, Alex M. Wong ${ }^{1}$, Ho-Ling Liu ${ }^{1,2}$, Yung-Liang Wan ${ }^{1}$, Yu-Chun Lin ${ }^{1,2}$, \\ An-Shine Chao ${ }^{3}$, Yao-Lung Chang ${ }^{3}$
}

Background: Prenatal magnetic resonance (MR) imaging demonstration of the normal spinal cord and the conus medullaris location has not been well studied. We compared balanced fast field echo (bFFE) with single-shot turbo spin-echo (SSh-TSE) MR sequences for visualizing the normal spinal cord and position of conus medullaris in fetuses.

Methods: This retrospective study was approved by the Institutional Review Board of Chang Gung Medical Foundation. We reviewed the MR images of 141 fetuses aged between 16 and 39 gestational weeks, to determine the position of the conus and visualize the spinal cord by using a signal intensity ratio of cerebral spinal fluid (CSF) to the spinal cord.

Results: Of the 75 subjects having normal spinal cord and being examined by both bFFE and SSh-TSE studies, the signal intensity ratio of $\mathrm{CSF} /$ cord was greater on bFFE images $(2.18 \pm 0.53)$ than that on SSh-TSE images $(1.21 \pm 0.13)(p<0.05)$. The conus level identified in the 50 subjects, in whom the lumbosacral spine was appropriately imaged, was located from L1 to L5 levels. The ascendance of the conus correlated moderately with

At a Glance Commentary

Scientific background of the subject

Gradient echo sequence imaging was superior in evaluating the fetal brain as compared with spin echo imaging. The location of the conus medullaris is essential to the diagnosis of spinal anomaly. The intrauterine MRI of the normal spinal cord and the position of conus have not been reported.

\section{What this study adds to the field}

We compared the efficacy of two fetal MRI sequence, bFFE and SSh-TSE, in fetuses with normal spinal cord, and concluded that bFFE sequence has better visualization of spinal cord. The location of fetal conus medullaris, which has not been studied with in vivo MRI, was identified and correlated with GA. gestational age.

Conclusions: With greater signal contrast ratio of CSF to spinal cord, bFFE sequence, when compared with SSh-TSE sequence, provides better visualization of normal spinal cord. The fetal conus medullaris ascends from L5 to L1 levels as the gestational age increases. (Biomed J 2014;37:232-236)

Key words: conus medullaris, fetal magnetic resonance imaging, spinal cord

$\mathrm{F}_{\mathrm{to}}^{\mathrm{cta}}$ etal magnetic resonance (MR) imaging has been shown - to have higher contrast resolution than ultrasonography (US) and gives better demonstration of the anatomy of the fetal brain. ${ }^{[1]}$ Balanced fast field echo (bFFE) sequence, which is a gradient-echo sequence, and single-shot turbo spin-echo (SSh-TSE) sequence are two commonly used fast T2-weighted fetal MR imaging techniques. Gradient-echo sequence imaging, when compared with spin-echo sequence

From the ${ }^{1}$ Department of Medical Imaging and Intervention, Chang Gung Memorial Hospital at Keelung and Linkou, Chang Gung University College of Medicine, Taoyuan, Taiwan; ${ }^{2}$ Department of Medical Imaging and Radiological Sciences, Chang Gung University, Taoyuan, Taiwan; ${ }^{3}$ Department of Obstetric and Gynecology, Chang Gung Memorial Hospital at Taoyuan, Chang Gung University College of Medicine, Taoyuan, Taiwan

Received: Mar. 02, 2013; Accepted: Sep. 05, 2013

Correspondence to: Dr. Alex M. Wong, Department of Medical Imaging and Intervention, Chang Gung Memorial Hospital at Linkou. 5 Fu-Hsing Street, Taoyuan, 333 Taiwan (R.O.C.). Tel: 886-3-3281200 ext. 2575; Fax: 886-3-3971936; E-mail: alexmcwchop@yahoo.com DOI: $10.4103 / 2319-4170.125649$ 
imaging, has been shown to have lower radiofrequency resorption and superior imaging quality in delineating myelination of the fetal brain. ${ }^{[2]}$

The location of the conus medullaris, which changes during the intrauterine life, is essential to the accurate diagnosis of spinal anomaly. The level of spinal cord termination during fetal life using MR imaging has not been well studied. A recent study using postmortem MR imaging reported that the conus laid between L2 and L5 levels before the $35^{\text {th }}$ gestational week and between L1 and L3 levels after the $35^{\text {th }}$ gestational week. ${ }^{[3]}$ However, intrauterine MR imaging of the normal spinal cord and the position of the conus at different gestational ages (GAs) have not been reported.

In this study, we compared the contrast resolution between bFFE imaging and SSh-TSE imaging in visualizing the spinal cord and determined the position of the conus in the fetuses. We hypothesized that the known conus ascendance that occurs with increasing GA could be identified by T2 MR images and that bFFE imaging provided greater contrast resolution than did SSh-TSE imaging in the visualization of the spinal cord.

\section{METHODS}

\section{Patients}

We retrospectively reviewed images of 141 fetuses with a GA between 16 and 39 weeks, referred to our institution for MR examination from 2004 to 2010 . We excluded 9 fetal subjects with spinal abnormality ( 2 myelomeningocele and 7 sacrococcygeal teratoma), leaving 132 subjects, among whom bFFE MR imaging was performed in 131 subjects and SSh-TSE imaging in 110 [Figure 1A].

\section{MR imaging}

All images were acquired on a $1.5 \mathrm{~T}$ scanner (Intera,
Philips Medical Systems, Best, the Netherlands) using a body coil. Images in sagittal, coronal, and transverse planes focusing on CNS system were obtained with bFFE [repetition time/ echo time of $4.4 \mathrm{~ms} / 2.2 \mathrm{~ms}$, flip angle of 80 , slice thickness of $4 \mathrm{~mm}$ without gap, field of vision (FOV) of 200-300 mm, and matrix of $256 \times 256$ ], SSh-TSE (repetition time/echo time of $600-800 \mathrm{~ms} / 60 \mathrm{~ms}$, flip angle of 90 , slice thickness of $4 \mathrm{~mm}$ without gap, FOV of $260-300 \mathrm{~mm}$, and matrix of $256 \times 256$ ), or both. Sagittal imaging focusing on the lumbosacral spine was performed in 69 fetuses, among whom 66 had bFFE imaging and 59 had SSh-TSE imaging [Figure 1B].

\section{Image interpretation}

\section{CSF to spinal cord signal intensity ratio determination}

Two investigators, a training radiologist with 3-year experience of fetal MR imaging and a neuroradiologist with 10-year experience of fetal MR imaging, interpreted the bFFE and SSh-TSE images of each subject to determine the visualization of the spinal cord. In subjects with visible spinal cord, a signal intensity ratio of the cerebral spinal fluid (CSF) to the spinal cord, representing an objective value of cord visualization, was measured. We assessed the signal intensity of the most clearly visualized part of the spinal cord and the signal intensity of the CSF in the thecal sac close to the measured spinal cord [Figure 2]. The region of interest (ROI) placed in the CSF and spinal cord in the same individual was constant, but varied among different subjects, in the range of $15-40 \mathrm{~mm}^{2}$. Of the two different techniques, the ROI was placed in the same or closest sagittal plane.

\section{Level of conus medullaris determination}

The position of the conus medullaris was determined with reference to the level of the lumbosacral transition. The

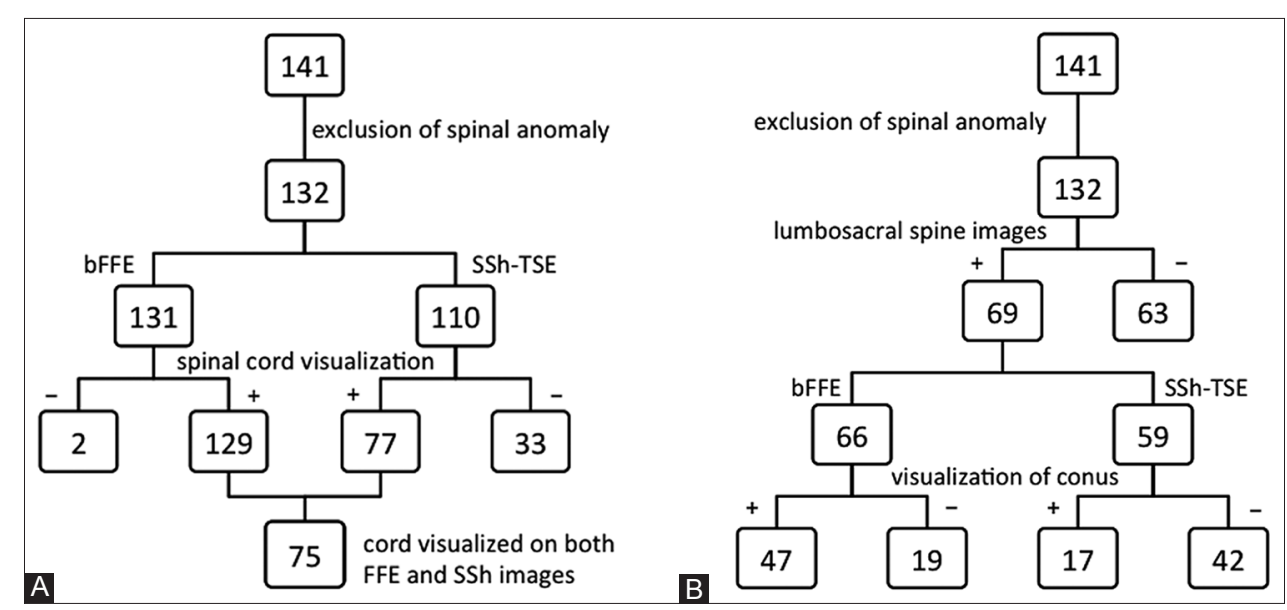

Figure 1: Flowchart for patient selection. (A) Selection of subjects for determination of CSF/cord signal intensity ratio on bFFE and SSh-TSE imaging. (B) Selection of subjects with lumbosacral spinal imaging to determine the position of conus. 
level of the conus medullaris of each subject was defined as the transition between the visualized spinal cord and CSF in the thecal sac, and the level was determined by referring to the sagittal bFFE or SSh-TSE images, on which the conus was most clearly identified.

\section{Statistical analysis}

Paired $t$-test was applied to determine the significant difference of the signal ratio of CSF to spinal cord between bFFE and SSh-TSE images on those subjects who were studied with both sequences. Pearson's correlation test was used to determine the relationship between the GA and the conus level. A $p$ value less than 0.05 was considered to indicate statistical significance. Analyses were performed by using a statistical software (SPSS, version 17; SAS Institute, Chicago, IL, U.S.A.).

\section{RESULTS}

\section{CSF to spinal cord signal intensity ratio determination}

In the 131 subjects with bFFE images, the spinal cord could be identified in 129 (98.47\%), while in the 110 subjects with SSh-TSE images, the cord could be identified in $77(70.0 \%)$ [Figure 1A]. In the 75 subjects with identifiable spinal cord who underwent both bFFE and SSh-TSE imaging, the signal intensity ratio of CSF to spinal cord was greater on bFFE images (mean: 2.18, standard deviation: 0.53) than on SSh-TSE images (mean: 1.21, standard deviation: 0.13) $(p<0.001)$.

\section{Level of conus medullaris determination}

The conus medullaris was identified in 47 fetuses (71.21\%; median GA: 26 weeks, range: $20-38$ weeks) on bFFE imaging and in 17 fetuses $(28.81 \%$; median GA: 30 weeks, range: 23-38 weeks) on SSh-TSE [Figure 1B]. Overall, the conus was identified in 50 subjects having either bFFE or SSh-TSE imaging, and the conus was located from L1 to L5 levels [Figures 3 and 4]. The ascendance of the conus was moderately and positively correlated with the GA in weeks $(r=0.66, p<0.05)$ [Figure 5].

\section{DISCUSSION}

We found a higher visualization rate of both of the spinal cord and conus medullaris on bFFE $(98.47 \%$ and $71.21 \%$, respectively) than on SSh-TSE (70.00\% and $28.81 \%$, respectively) and a significantly higher signal ratio of CSF to spinal cord on bFFE $(2.18 \pm 0.53)$ than on SSh-TSE $(1.21 \pm 0.13)$. Better visualization can be explained by the greater signal contrast between CSF and spinal cord on bFFE than on SSh-TSE and by blurring of tissue having short T2 relaxation

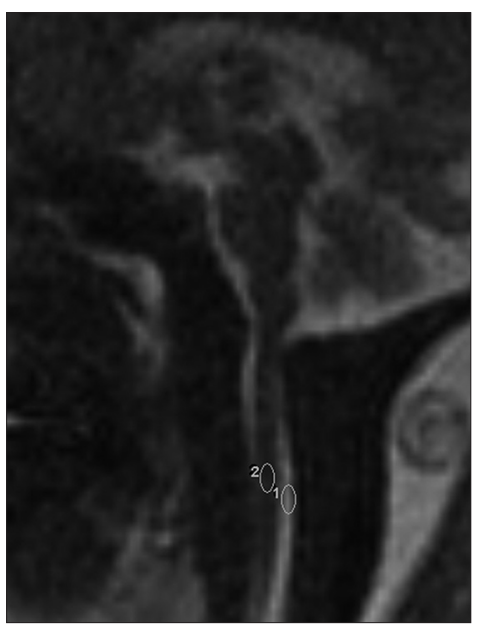

Figure 2: Demonstration of signal intensity ratio measurement. On a sagittal bFFE image of a $32^{\text {nd }}$ gestational week fetus, ROIs were placed over the cervical spinal cord and the CSF in the adjacent thecal sac.

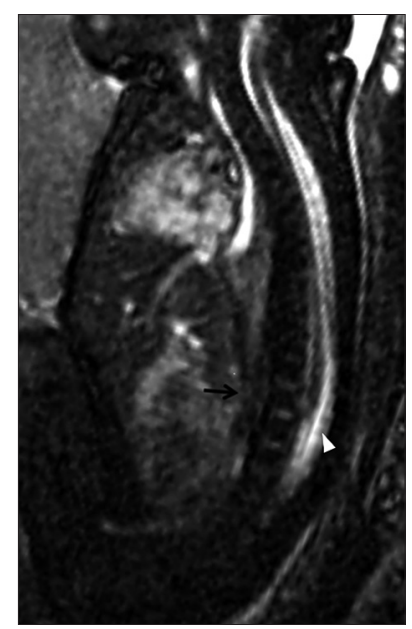

Figure 3: Sagittal bFFE image of a fetus at $27^{\text {th }}$ gestational week shows the relative position of T-L spine and spinal cord termination (arrowhead), which is located at L1 level (arrow).

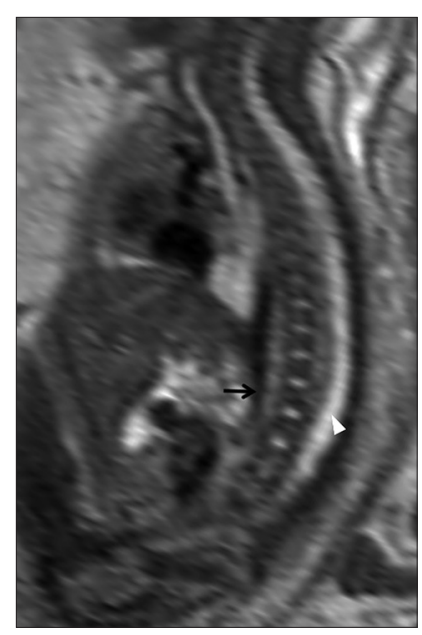

Figure 4: Sagittal SSh-TSE image of the same fetus at $27^{\text {th }}$ week shows the cord termination (arrowhead) at L1 level (arrow). 


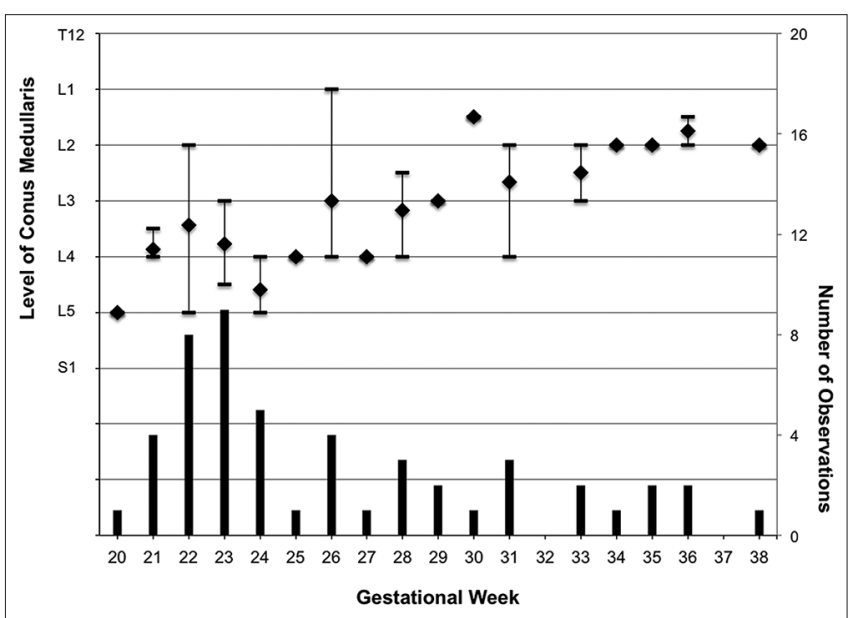

Figure 5: Range and mean of the location of fetal conus medullaris plotted against gestational weeks. The level of conus medullaris ascends from L5 to L1 levels as the GA increases from the $20^{\text {th }}$ to $38^{\text {th }}$ weeks. The number of observations related to each GA week is shown in the bar chart.

time (e.g., the spinal cord) on TSE sequence. The MR signal of bFFE can be expressed as signal $=\mathrm{M}_{0} \cdot \sin \alpha /[1+\cos \alpha$ $+(1-\cos \alpha)(\mathrm{T} 1 / \mathrm{T} 2)]$, where $\mathrm{M}_{0}$ is the magnetization at thermal equilibrium and $\alpha$ is the flip angle, ${ }^{[4]}$ and the signal of SSh-TSE can be estimated as signal $=\mathrm{M}_{0}$. $\exp (-\mathrm{TE} / \mathrm{T} 2){ }^{[5]}$ By substituting the mean relaxation times and relative proton density ratio of CSF $(\mathrm{T} 1=3916 \mathrm{~ms}, \mathrm{~T} 2=1330 \mathrm{~ms} ; 1.09)$ and gray matter $(\mathrm{T} 1=1213 \mathrm{~ms}, \mathrm{~T} 2=126 \mathrm{~ms} ; 1.0)$ recently reported by Weigel et al. ${ }^{[5]}$ the theoretical contrast ratio of CSF to gray matter in our study can be estimated to be 2.76 for bFFE $\left(\alpha=80^{\circ}\right)$ and 1.68 for SSTSE $(\mathrm{TE}=60 \mathrm{~ms}$ ), comparable to the results in our study.

Decreased visualization of the spinal cord may be associated with blurring in SSh-TSE. Point spread function blurring, caused by continuous T2 decay in SSh-TSE owing to data of the outer K-space being acquired from late echoes, ${ }^{[2]}$ has been known to occur in tissues with short $\mathrm{T} 2$ relaxation time. Since the spinal cord has shorter T2 relaxation time than the CSF, blurring may occur at the interface between the cord and the surrounding CSF, leading to a decrease in both the signal contrast and the visualization of the spinal cord.

The level of termination of the spinal cord was reported to be about L5 at the $20^{\text {th }}$ week of gestation and to reach about L1 within the first year of life in a postmortem study. ${ }^{[6,7]} \mathrm{In}$ a recent MR imaging study of the fetal lumbar spine, also a postmortem study, the level of cord termination was found between L2 and L5 levels in fetuses younger than 35 gestational weeks and between L1/2 and L2/3 levels in fetuses near term. ${ }^{[3]}$ On postnatal sonography, the position of the conus medullaris was found at the $\mathrm{L} 2 / 3$ level in premature babies from $27^{\text {th }}$ to $29^{\text {th }}$ gestational week and at the L1/2 level in babies older than $34^{\text {th }}$ gestational week. ${ }^{[8]}$ A recent intrauterine US study of 110 fetuses reported that the conus medullaris ascended between the $13^{\text {th }}$ and $40^{\text {th }}$ gestational weeks from below the L4 vertebral level to L2 level and above ${ }^{[9]}$ Another fetal US study reported the conus positions within L2-L3 levels in fetuses from $20^{\text {th }}$ to $24^{\text {th }}$ gestational weeks. ${ }^{[10]}$ Our study results, showing the mean conus position at the $\mathrm{L} 4$ level in fetuses between $21^{\text {st }}$ and $25^{\text {th }}$ gestational weeks, at L3/4 level in fetuses between $26^{\text {th }}$ and $30^{\text {th }}$ weeks, at $\mathrm{L} 2 / 3$ level in fetuses between $31^{\text {st }}$ and $35^{\text {th }}$ weeks, and at L2 level in fetuses older than $36^{\text {th }}$ week, were more comparable to those of the postmortem fetal and postnatal studies. The conus medullaris was found in several normal fetuses at as low as the L4 level between 25 and 31 weeks GA, and was consistently observed at the L3 level or higher after 31 weeks GA.

Compared with the postmortem studies of the fetal spine, our study was an in vivo one and without fixation damage or autolysis of tissue, thus could be more clinically applicable. Images in the sagittal plane were available in all of our subjects recruited for determining the level of spinal cord termination.

Our study has a few limitations. The age distribution of the subjects was uneven with fewer subjects imaged in the third trimester, reflecting the clinical indications for the fetal MR examinations in the second trimester for decision making in managing pregnancy. Future study may employ a larger population involving more subjects in late GAs. Although nearly half of our cases were specifically examined for the lumbosacral spine with sagittal images, only a small number of subjects had both the conus and the lumbosacral transition imaged on a single sagittal image, which may affect localization of the vertebral level. Since lumbosacral transitional vertebrae may be present in $4-21 \%$ of the general population, ${ }^{[11,12]}$ variations may exist in identifying the vertebral level, a process that depends on morphological recognition of the lumbosacral junction.

\section{Conclusions}

In summary, fetal spinal MR imaging using the bFFE sequence, when compared with the SSh-TSE sequence, has greater signal contrast ratio of CSF to spinal cord and provides better visualization of the spinal cord. The level of spinal cord termination ascends from L5 to L1 levels as the GA increases from the $20^{\text {th }}$ to $38^{\text {th }}$ weeks as observed with prenatal ultrasound.

\section{REFERENCES}

1. Coakley FV, Glenn OA, Qayyum A, Barkovich AJ, Goldstein R, Filly RA. Fetal MRI: A developing technique for the developing patient. AJR Am J Roentgenol 2004;182:243-52.

2. Chung HW, Chen CY, Zimmerman RA, Lee KW, Lee CC, Chin SC. T2-weighted fast MR imaging with true FISP versus HASTE: 
Comparative efficacy in the evaluation of normal fetal brain maturation. AJR Am J Roentgenol 2000;175:1375-80.

3. Widjaja E, Whitby EH, Paley MN, Griffiths PD. Normal fetal lumbar spine on postmortem MR imaging. AJR Am J Neuroradiol 2006;27:553-9.

4. Huang TY, Huang IJ, Chen CY, Scheffler K, Chung HW, Cheng HC. Are TrueFISP imagesT2/T1-weighted? Magn Reson Med 2002;48:684-8.

5. Weigel M, Hennig J. Contrast behavior and relaxation effects of conventional and hyperecho-turbo spin echo sequences at 1.5 and 3 T. Magn Reson Med 2006;55:826-35.

6. Barson A. The vertebral level of termination of the spinal cord during normal and abnormal development. J Anat 1970;106:489-97.

7. Govender S, Charles RW, Haffejee MR. Level of termination of the spinal cord during normal and abnormal fetal development. S Afr Med J 1989;75:484-7.
8. Beek FJ, de Vries LS, Gerards LJ, Mali WP. Sonographic determination of the position of the conus medullaris in premature and term infants. Neuroradiology 1996;38(Suppl 1):S174-7.

9. Zalel Y, Lehavi O, Aizenstein O, Achiron R. Development of the fetal spinal cord-Time of ascendance of the normal conus medullaris as detected by sonography. J Ultrasound Med 2006;25:1397-401.

10. Perlitz Y, Izhaki I, Ben-Ami M. Sonographic evaluation of the fetal conus medullaris at 20 to 24 weeksandapos; gestation. Prenat Diagn 2010;30:862-4.

11. O'Driscoll CM, Irwin A, Saifuddin A. Variations in morphology of the lumbosacral junction on sagittal MRI: Correlation with plain radiography. Skeletal Radiol 1996;25:225-30.

12. Tini PG, Wieser C, Zinn WM. The transitional vertebra of the lumbosacral spine: Its radiological classification, incidence, prevalence, and clinical significance. Rheumatol Rehabil 1977;16:180-5. 